\title{
Analysis of GeV-band gamma-ray emission from SNR RX J1713.7-3946
}

\author{
R. Brose*, S. Federici, M. Pohl, I. Telezhinsky, A. Wilhelm \\ Institute of Physics and Astronomy, University of Potsdam, Karl-Liebknecht-Strasse 24/25, \\ 14476 Potsdam, Germany \\ DESY, Platanenallee 6, 15738 Zeuthen, Germany \\ E-mail: robert.broseddesy.de

\section{V.V. Dwarkadas} \\ University of Chicago, Department of Astronomy\& Astrophysics, 5640 S Ellis Ave, TAAC 55, \\ Chicago, IL 60637, U.S.A.
}

\begin{abstract}
RX J1713-3946 is the brightest shell-type supernova remnant (SNR) of the TeV gamma-ray sky. Earlier Fermi-LAT results on low energy gamma-ray emission suggested that, despite large uncertainties in the background determination, the spectrum is inconsistent with a hadronic origin. We update the GeV-band spectra using improved estimates for the diffuse galactic gamma-ray emission and more than double the volume of data. We further investigate the viability of hadronic emission models for RX J1713-3946. We produced a high-resolution map of the diffuse Galactic gamma-ray background corrected for the HI self-absorption and used it in the analysis of more than five years worth of Fermi-LAT data. We used hydrodynamic scaling relations and a kinetic transport equation to calculate the acceleration and propagation of cosmic rays in SNR. We then determined spectra of hadronic gamma-ray emission from RX J1713-3946, separately for the SNR interior and the cosmic-ray precursor region of the forward shock, and computed flux variations that would allow us to test the model with observations. We find that RX J1713-3946 is now detected by Fermi-LAT with very high statistical significance, and the source morphology is best described by that seen in the TeV band. The measured spectrum of RX J1713-3946 is hard with index $\gamma=1.53 \pm 0.07$, and the integral flux above $500 \mathrm{MeV}$ is $F=(5.5 \pm 1.1) \times 10^{-9}$ photons $\mathrm{cm}^{-2} \mathrm{~s}^{-1}$. We demonstrate that scenarios based on hadronic emission from the cosmic-ray precursor region are acceptable for RX J1713-3946, and we predict a secular flux increase at a few hundred $\mathrm{GeV}$ at the level of around $15 \%$ over ten years, which may be detectable with the upcoming Cherenkov Telescope Array (CTA) observatory.
\end{abstract}

The 34th International Cosmic Ray Conference,

30 July- 6 August, 2015

The Hague, The Netherlands

\footnotetext{
* Speaker.
} 


\section{Introduction}

RX J1713.7-3946, also known as G347.3-0.5, is a young ( $t_{\mathrm{SNR}} \simeq 1600 \mathrm{yr}$ ) shell-type SNR located in the Galactic plane. Observations with the Japanese ASCA satellite [1] showed a featureless $\mathrm{X}$-ray spectrum in the northwest shell of the remnant, clearly indicating nonthermal emission. Later ASCA observations [2] confirmed the absence of line emission and demonstrated that the X-ray spectra of all parts of the remnant are power laws with indices between $\sim 2.2$ and 2.4. More recent studies conducted with Chandra [3] and XMM-Newton [4] satellites clearly showed small-scale spatial variations of the photon index ranging from 1.8 to 2.6, which are tracers of the acceleration and propagation history of recently accelerated electrons [5].

At higher energies two competing radiation processes can produce a similar flux in the GeV$\mathrm{TeV}$ energy range. In the leptonic scenario, $\gamma$-rays are produced by electrons via inverse Compton scattering, and in the hadronic scenario $\gamma$-rays are due to $\pi^{0}$-decay from proton-proton interactions. The H.E.S.S. telescope array detected RX J1713.7-3946 at the TeV-scale [6] and showed a similarity between the X-ray and TeV-band morphologies. The Large Area Telescope (LAT) [7] measured extended GeV $\gamma$-ray emission coincident with the position of RX J1713.7-3946 [8]. The spectral analysis found a very hard spectrum (photon index $\gamma \simeq 1.5$ ), which was well in agreement with a leptonic scenario. Nonetheless, this conclusion did not exclude that protons are accelerated in this SNR.

In this paper, we reanalyze Fermi-LAT data that have more than doubled in exposure since the original publication. Moreover, we develop dedicated tools to produce high-resolution background maps that take HI self-absorption into account. Our background modeling permits determining the key parameters of $\gamma$-ray emission from RX J1713.7-3946 with reduced systematic uncertainty. Next, we present a model for hadronic $\gamma$-ray emission from the cosmic-ray precursor of the forward shock of RX J1713.7-3946, which is in principle viable, and, what is more important, we propose a way to verify the model. We predict a $\gamma$-rayflux increase at the level of $15 \%$ on a timescale of ten years and suggest that this flux variation might be detectable with the Cherenkov Telescope Array (CTA) [9]. A full report on our study and its results is published elsewhere [10].

\section{Analysis and results}

The likelihood analysis of data from Fermi-LAT requires, among others, a template for diffuse Galactic $\gamma$-ray emission, which is based on infrared tracers and spectral line information on atomic hydrogen and carbon monoxide as tracer of molecular gas. While the absolute normalization of the templates is adjustable in the likelihood optimization, its angular structure is not. If an extended source is to be analyzed, a poor representation of the diffuse background will lead to systematic errors in the reconstructed morphology and possibly a significant offset in the derived spectrum of the source.

Where available, we have used data from the Southern Galactic Plane Survey [11] to refine the standard model of galactic diffuse emission to a higher intrinsic angular resolution than is provided in single-dish HI surveys, and to correct for HISA using the algorithm described in [12]. The HI column density derived for the absorber and that associated with the absorption correction to the original line signal are placed on the line of sight using the method of [13]. We then use the 


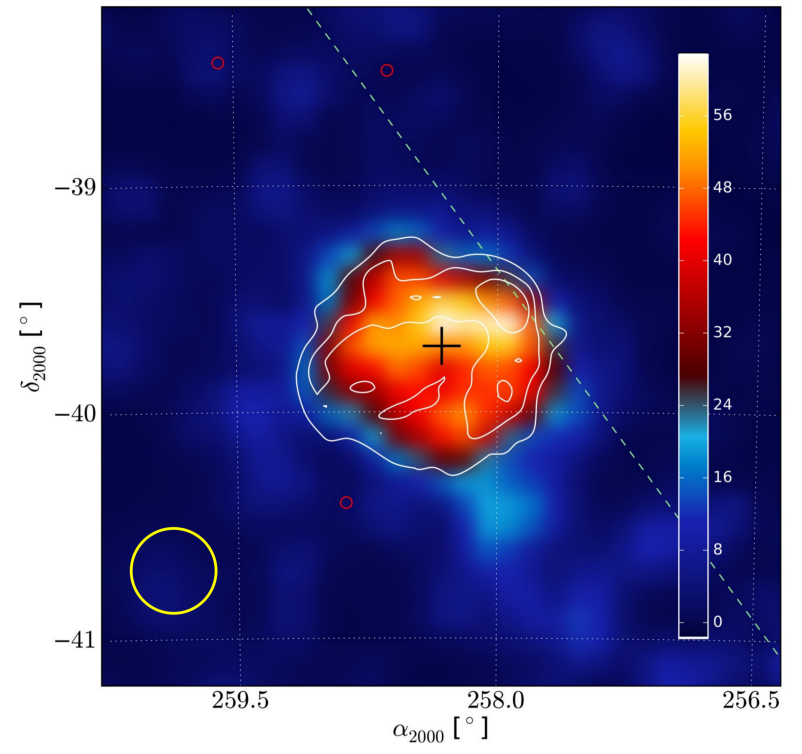

Figure 1: Map of the test statistic (TS) for a point-like source in the region around RX J1713.7-3946. The black cross denotes the best-fit centroid of a disk model and the yellow circle indicates the energy-averaged $68 \%$ confinement region of the point-spread function. Red circles indicate the position of the second Fermi-LAT catalog sources in the background model. Shown in white are contours of gamma-ray excess counts based on H.E.S.S. observations, the levels are 25, 50, and 75. Figure taken from [10], reproduced with permission, (c)ESO.

gamma-ray emissivity derived with the GALPROP code, version 54, with best-fit parameters given in [14], to compute the expected $\gamma$-ray intensity from the additional HI column density, which we found in the HISA search. Finally, we correct the standard templates of diffuse galactic $\gamma$-ray emission for the additional signal. The data analysis is done using the publicly available FermiLAT science tools, version 9.31.1, and the post-launch P7v6 data selection with the appropriate instrument response functions.

\subsection{Position and spatial extension of RX J1713.7-3946}

We first need to find the localization and spatial distribution of emission from RX J1713.73946. For that purpose, we construct a map of test statistic (TS) [15]), defined as logarithmic improvement in the likelihood function $L, \mathrm{TS}=2\left(\ln L-\ln L_{0}\right)$, on a grid of locations with $0.1^{\circ}$ spacing. At each point of the grid the parameters of the point-like source are free to vary and those of all background components are fixed at the values found in a global likelihood analysis. The envelope of signal with TS $\geq 36$ in the resulting TS map, shown in Fig. 1, suggests spatially extended emission.

To find the best position of the source and hence minimize the systematic uncertainties, we then modeled RX J1713.7-3946 as a uniform disk with a radius of $0.5^{\circ}$. The best-fit centroid for the disk model is indicated in Fig 1 and located at $\alpha=258.32^{\circ}$ and $\delta=-39.71^{\circ}$ in J2000 with an error radius of $0.02^{\circ}$ at the $68 \%$ confidence level. All the following analyses are performed using spatial templates centered at this best-fit position.

To investigate the spatial morphology of the emission associated with RX J1713.7-3946, a number of spatial templates are tested, five of which are uniform disks with radii ranging from $0.5^{\circ}$ to $0.7^{\circ}$. An additional template is designed to reflect the $\mathrm{TeV}$-band intensity distribution observed with the H.E.S.S. telescope. Table 1 summarizes the best-fit parameters in the likelihood analysis of each template, performed in the energy range from $500 \mathrm{MeV}$ to $300 \mathrm{GeV}$ by optimizing a spectral 


\begin{tabular}{|l|c|c|c|}
\hline Morphology & Flux & Photon index & TS \\
\hline Disk $r=0.50^{\circ}$ & $4.982 \pm 0.916$ & $1.508 \pm 0.075$ & 160 \\
Disk $r=0.55^{\circ}$ & $5.122 \pm 0.941$ & $1.509 \pm 0.075$ & 161 \\
Disk $r=0.60^{\circ}$ & $5.449 \pm 1.069$ & $1.513 \pm 0.075$ & 159 \\
Disk $r=0.65^{\circ}$ & $5.547 \pm 1.052$ & $1.514 \pm 0.075$ & 156 \\
Disk $r=0.70^{\circ}$ & $5.908 \pm 1.216$ & $1.521 \pm 0.076$ & 150 \\
H.E.S.S. & $5.522 \pm 1.075$ & $1.528 \pm 0.074$ & 163 \\
\hline
\end{tabular}

Table 1: Morphological analysis of the $\gamma$-ray emission associated with RX J1713.7-3946. The integral flux from RX $\mathrm{J} 1713.7-3946$ is calculated over the energy range $500 \mathrm{MeV}-300 \mathrm{GeV}$ and it is given in units of $10^{-9}$ photons $\mathrm{cm}^{-2} \mathrm{~s}^{-1}$.

model of the form

$$
\frac{d N}{d E}=N_{0}\left(\frac{E}{E_{0}}\right)^{-\gamma}
$$

where $N_{0}$ is the prefactor, $E_{0}$ the energy scale, and $\gamma$ is the spectral index.

All models yield a high test statistic $(150 \leq \mathrm{TS} \leq 163)$, about three times that achieved in a point-source fit, implying that the emission is well resolved by the LAT as an extended region. For uniform-disk templates, TS slowly decreases beyond a radius of $0.55^{\circ}$, indicating that this value marks the extent of GeV-scale emission from RX J1713.7-3946. The H.E.S.S. template fits best, and we use the H.E.S.S. template, centered at $\alpha=258.32^{\circ}$ and $\delta=-39.71^{\circ}$ in $\mathrm{J} 2000$, in the following spectral analysis. In the end, the vagaries in choosing the spatial template and centroid position contribute to the systematic-uncertainty margin that we shall discuss below.

\subsection{Energy spectrum of RX J1713.7-3946}

First, we perform a global likelihood analysis using the H.E.S.S. template as spatial model for RX J1713.7-3946. The fit yields $\gamma=1.53 \pm 0.07$, and the measured integral photon flux above 500 $\mathrm{MeV}$ is $F=(5.52 \pm 1.07) \times 10^{-9}$ photons $\mathrm{cm}^{-2} \mathrm{~s}^{-1}$.

Then, the entire energy range is divided into nine logarithmically spaced energy bins, in each of which a likelihood fit is performed using the H.E.S.S. template as spatial model for RX J1713.73946 and the spectral model and parameters obtained in the global fit (and shown in Table 1). The resulting energy spectrum of RX J1713.7-3946 is shown in Fig. 2 together with previously published H.E.S.S. measurements [16]. The nine Fermi-LAT data points (black crosses) only include statistical errors. The dotted line at the bottom of the histogram denotes the threshold (TS $=7)$ for setting a 95\% confidence-level upper limit on the Fermi-LAT data points. The physical interpretation of the SED will be discussed in the next section.

\section{Interpretation}

The hard spectrum of the GeV-band emission initially discovered by [8] and confirmed here suggests an inverse-Compton origin of the emission. In a simple one-zone scenario for the particle distribution and subsequent emission, it is difficult to accommodate a strong hadronic component. In this section, we point out that more complex scenarios are possible, although they require some degree of fine-tuning.

RX J1713.7-3946 was likely produced by a core-collapse supernova. The progenitor star would have emitted a wind that pushed the ambient medium into a dense gas shell at some dis- 


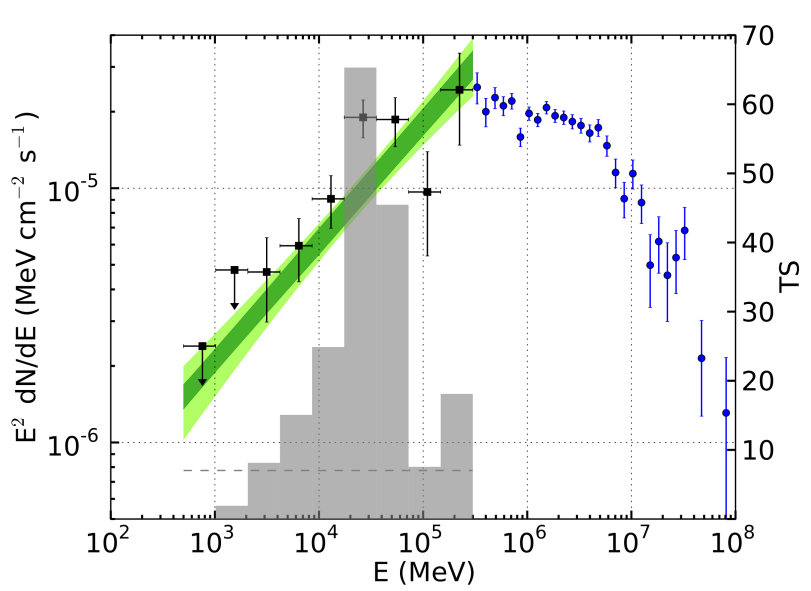

Figure 2: Broadband $\gamma$-ray spectrum of RX J1713.7-3946 as measured here (black points) and by H.E.S.S. (blue points). The two bow-tie bands denote systematic (dark green) and total (light green) uncertainties of the single power-law fit, the latter assuming that systematic and statistical uncertainties can be added in quadrature. The histogram indicates the TS value for each energy bin. The dotted line is the threshold for setting a 95\% confident level upper limit. Figure taken from [10], reproduced with permission, CCESO.

tance. The high velocity currently measured suggests that the forward shock has not yet reached the wall enclosing the wind-blown cavity.

Dense gas clouds may survive passage through the forward shock of SNRs [17]. Slow and energy-dependent diffusion of cosmic rays into these clouds would then lead to a hard particle spectrum at the high-density core of the cloud. A fit to the $\gamma$-ray spectrum requires a total cloud mass of $\sim 500 M_{\odot}$ inside the SNR [18]. This is much more than can condense out of the wind of the massive progenitor star, and so the scenario would require that pre-existing clouds have been unaffected by the wind. Besides the fact that nonpenetration of clouds is not observed in the $\gamma$-ray spectra of nearby molecular complexes [19], we see two difficulties with this scenario.

Firstly, the medium around massive stars tends to be largely homogenized before they explode as core-collapse supernova, owing to photoevaporation and the rocket effect [20]. Secondly, as the stellar wind streams around dense gas clouds, Kelvin-Helmholtz instabilities, followed by Rayleigh-Taylor instabilities will disrupt and wash off their outer layers, leading to large streams of gas with moderate density $\left(n \approx 1-10 \mathrm{~cm}^{-3}\right)$ that upon contact with the SNR forward shock would emit intense X-ray emission. One would have to demonstrate that its intensity is compatible with the published upper limits for thermal X radiation.

A large fraction of the gas clouds and the outer gas shell will thus be located outside of the SNR, where they would be illuminated by the cosmic-ray precursor to the forward shock. The width of the cosmic-ray precursor to astrophysical shocks is strongly energy dependent on account of the momentum dependence of the mean free path for scattering. At a given location, this leads to very hard particle spectra and, if target gas is present, correspondiongly hard $\gamma$-ray spectra with cutoff at an energy determined by the distance from the shock, the diffusion coefficient, and the shock velocity. In the case of RX J1713.7-3946, the target gas has to be located very close to the forward shock, otherwise we cannot observe intense $\gamma$-ray emission at 50-500 GeV. Consequently, it is illuminated not primarily by so-called runaway cosmic rays that have escaped from the system, but by the cosmic-ray precursor to the forward shock. We predict a gradual but detectable increase in $\gamma$-ray flux over a decade or two that renders this scenario testable. To substantiate our claim, we first present an analytic estimate to illustrate why the signal would grow with time. We then use a more sophisticated model to calculate the time dependence of the $\gamma$-ray spectrum.

Suppose a gas cloud is located at radius $r_{0}$, corresponding to the time-dependent comoving 
coordinate $\rho_{0}(t)=r_{0}-r_{\mathrm{s}}(t) \simeq r_{0}-\dot{r}_{\mathrm{s}} t$, where the last expression is approximately valid for short time intervals. The gas cloud is illuminated with cosmic rays of differential density,

$$
N \simeq N_{0}(E) \exp \left(\int^{\rho_{0}} d \rho^{\prime} \frac{V_{\rho}}{D\left(E, \rho^{\prime}\right)}\right) .
$$

The timescale on which the cosmic-ray illumination changes can be estimated as

$$
\tau \simeq \frac{N}{\left|\frac{\partial N}{\partial t}\right|} \simeq \frac{D\left(E, \rho_{0}\right)}{\left|\dot{\rho}_{0} V_{\rho}\right|} \simeq \frac{D\left(E, \rho_{0}\right)}{\dot{r}_{\mathrm{s}}^{2}}
$$

If we scale the diffusion coefficient to the Bohm limit, $D=\eta r_{\mathrm{L}} c / 3$, we find for the timescale of variation

$$
\tau \simeq(10 \mathrm{yrs}) \eta\left(\frac{E}{10 \mathrm{TeV}}\right)\left(\frac{B}{10 \mu \mathrm{G}}\right)^{-1}\left(\frac{\dot{r}_{\mathrm{s}}}{3000 \mathrm{~km} / \mathrm{s}}\right)^{-2}
$$

Protons with $10 \mathrm{TeV}$ in kinetic energy produce $\gamma$-rays at energies around $500 \mathrm{GeV}$. As long as the diffusion coefficient is not significantly larger than the Bohm limit, we expect observable variations in the $\gamma$-ray flux from bright, young SNRs such as RX J1713.7-3946, in particular if streaming instabilities amplify the magnetic field to amplitudes in excess of $10 \mu \mathrm{G}$. To be noted is that the variation timescale does not explicitly depend on the distance from the shock. Implicitly, a certain distance dependence arises from the fact that the diffusion coefficient must be evaluated near the location of the gas cloud, not at the shock.

For detailed modeling, we consider a system consisting of a core-collapse SNR and a shell of dense gas located just outside the remnant. We numerically solve the full transport equation for cosmic rays on a grid that extends from the SNR center to several dozens of SNR radii ahead of the forward shock (For details see [21, 22, 23]). The grid extension allows us to account for particle escape from the precursor. We can use either full hydrodynamical simulations or analytic solutions to trace the evolution of the SNR. The diffusion coefficient is assumed to be Bohm-like at the shock, $D=r_{L} v / 3$. Ahead of the shock the diffusion coefficient linearly increases [24, 25], until thirty SNR radii ahead of the shock it reaches $D_{G}=10^{29}(E / 10 \mathrm{GeV})^{1 / 3}(B / 3 \mu \mathrm{G})^{-1 / 3}$, a value typical for the galactic propagation. Close to the shock a typical cosmic-ray precursor will be established. Further ahead, the probability of escape from the system becomes significant, and particles may leave the system.

We used analytical solutions for the free-expansion stage of SNR expanding into a wind-blown cavity [26] at the fiducial age 1600 years. The current SNR radius, $R \simeq 8.65 \mathrm{pc}$, and shock speed, $V_{s h} \simeq 4200 \mathrm{~km} / \mathrm{s}$, (for a distance of $1 \mathrm{kpc}$ ) permit a range of parameter values for the mass-loss rate and the progenitor wind speed. As an example, we assumed for the mass-loss rate $\dot{M} \simeq 10^{-4} M_{\odot}$, for the wind speed $v_{\mathrm{w}} \simeq 50 \mathrm{~km} / \mathrm{s}$, and for the explosion energy $E_{S N}=10^{51} \mathrm{ergs}$, corresponding to a relatively fast red-supergiant wind. It serves as demonstration of the stability of our results against the choice of hydrodynamical parameters that even for a Sedov solution the flux increase at a few $100 \mathrm{GeV}$ was virtually the same. The magnetic field in the precursor region is assumed to be $B_{p}=23 \mu \mathrm{G}$, which is shock-compressed to a downstream field $B_{d}=75 \mu \mathrm{G}$ inside the SNR $[27,28]$.

The location of the dense gas shell is prescribed by the necessity to reproduce the presently observed $\gamma$-ray spectrum from RX J1713.7-3946, as the precursor of cosmic rays at $10 \mathrm{TeV}$ should 


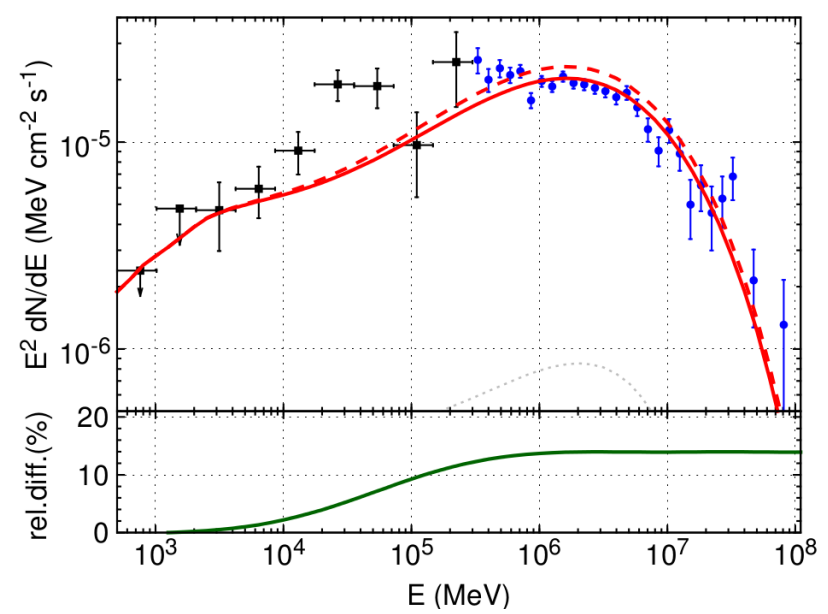

Figure 3: Broadband $\gamma$-ray spectrum of RX J1713.7-3946 as measured with LAT (our results, black points) and with H.E.S.S. (blue points), compared to calculated HE $\gamma$-ray spectra at the age of 1600 years (solid red line) and 1610 years (dashed red line). The IC contribution to both spectra is indicated for additional information (gray dotted line). The relative difference between spectra at two epochs is shown in the lower panel (solid green line). Figure taken from [10], reproduced with permission, (C)ESO.

just reach it. That leaves the diffusion coefficient and, in particular, the efficacy of magnetic-field amplification as the main sources of uncertainty, for which we use conservative assumptions.

We then calculate emission spectra and intensity maps at two epochs, at 1600 and at 1610 years, including pion-decay [29], inverse Compton (IC), and synchrotron emission in turbulent magnetic field [30]. The results of our calculations are presented at Fig. 3 (solid red line). The HE $\gamma$-ray spectrum at the age of 1600 years is normalized to fit the H.E.S.S. measurements [16]. The numerical treatment indicates a flux increase of $15 \%$ over ten years, consistent with the analytical estimate. The main prediction of our model thus is a secular increase with time of the HE $\gamma$-ray flux that is largely independent of the choice of parameters, provided they permit reproducing the peak in the $v F_{v}$ spectrum at $\sim 1 \mathrm{TeV}$ for the current size and expansion rate of RX J1713.7-3946.

\section{Conclusions}

In this work we analyzed more than five years of Fermi-LAT observations of the SNR RX J1713.7-3946, using high-resolution $\gamma$-ray background maps that account for HI self-absorption. RX J1713.7-3946 appears as circular source with radius $r \simeq 0.55^{\circ}$ that is detected at $13 \sigma$ level in the energy range between $500 \mathrm{MeV}$ and $300 \mathrm{GeV}$. We see a tendency in the $\mathrm{GeV}$ band for the same intensity enhancement in the northwest region of the remnant that is seen with H.E.S.S.. The measured spectrum of RX J1713.7-3946 shows a hard spectral index of $\gamma=1.53 \pm 0.07$ with an integral flux above $500 \mathrm{MeV}$ of $F=(5.5 \pm 1.1) \times 10^{-9}$ photons $\mathrm{cm}^{-2} \mathrm{~s}^{-1}$.

We introduced a model of hadronic emission from RX J1713.7-3946 that reproduces the hard $\mathrm{GeV}$ spectrum with index $\gamma \simeq 1.5$ as emission from a shell of dense gas that is located a short distance upstream of the forward shock. Although this hadronic scenario is somewhat speculative, it has the benefit of being testable: we predict the hadronic $\gamma$-ray flux to increase at the level of $15 \%$ over ten years, which should be observable with the future CTA facility that is projected to operate for more than ten years [9]. We have verified that the growth in $\gamma$-ray flux is largely independent of the choice of parameters, provided they reproduce a $v F_{v}$-peak at $\sim 1 \mathrm{TeV}$. Whereas our model assumes spherical symmetry, real SNR are not that simple, and conditions are not the same in different regions. The stability of our result suggests that such a variation of parameters does not significantly reduce the secular increase in $\gamma$-ray flux. 
Acknowledgements: Part of this work was supported by the Helmholtz Alliance for Astroparticle Physics HAP funded by the Initiative and Networking Fund of the Helmholtz Association. VVD's research on the high energy emission from young SNRs is supported by NASA Fermi grant NNX12A057G.

\section{References}

[1] Koyama, K., Kinugasa, K., Matsuzaki, K., et al. 1997, PASJ, 49, L7

[2] Slane, P., Gaensler, B. M., Dame, T., et al. 1999, ApJ, 525, 357

[3] Uchiyama, Y., Aharonian, F., \& Takahashi, T. 2003, A\&A, 400, 567

[4] Cassam-Chenaï, G., Decourchelle, A., Ballet, J., et al. 2004, A\&A, 427, 199

[5] Rettig, R. \& Pohl, M. 2012, A\&A, 545, A47

[6] Aharonian, F. et al. 2004, Nature, 432, 75

[7] Atwood, W. et al. 2009, ApJ, 697, 1071

[8] Abdo, A. A., Ackermann, M., Ajello, M., et al. 2011, ApJ, 734, 28

[9] Acharya, B. S., Actis, M., Aghajani, T., et al. 2013, Astroparticle Physics, 43, 3

[10] Federici, S., Pohl, M., Telezhinsky, I., Wilhelm, A., \& Dwarkadas, V. V. 2015, A\&A, 577, A12

[11] McClure-Griffiths, N. M., Dickey, J., Gaensler, B., et al. 2005, ApJ Suppl., 158, 178

[12] Gibson, S. J., Taylor, A. R., Higgs, L. A., Brunt, C. M., \& Dewdney, P. E. 2005, ApJ, 626, 214

[13] Pohl, M., Englmaier, P., \& Bissantz, N. 2008, ApJ, 677, 283

[14] Strong, A., Porter, T., Digel, S., et al. 2010, ApJ, 722, L58

[15] Mattox, J., Bertsch, D., Chiang, J., et al. 1996, ApJ, 461, 396

[16] Aharonian, F., Akhperjanian, A. G., Bazer-Bachi, A. R., et al. 2011, A\&A, 531, C1

[17] Inoue, T., Yamazaki, R., Inutsuka, S.-i., \& Fukui, Y. 2012, ApJ, 744, 71

[18] Gabici, S. \& Aharonian, F. A. 2014, MNRAS, 445, L70

[19] Ackermann, M., Ajello, M., Allafort, A., et al. 2012, ApJ, 755, 22

[20] McKee, C. F., van Buren, D., \& Lazareff, B. 1984, ApJ Lett., 278, L115

[21] Telezhinsky, I., Dwarkadas, V., \& Pohl, M. 2012, Astrop. Phys., 35, 300

[22] Telezhinsky, I., Dwarkadas, V. V., \& Pohl, M. 2012, A\&A, 541, A153

[23] Telezhinsky, I., Dwarkadas, V. V., \& Pohl, M. 2013, A\&A, 552, A102

[24] Bell, A. R. 1978, MNRAS, 182, 147

[25] Drury, L. O. 1983, Rep. Prog. Phys., 46, 973

[26] Truelove, J. K. \& McKee, C. F. 1999, ApJ Suppl., 120, 299

[27] Ballet, J. 2006, Adv. Sp. Res., 37, 1902

[28] Acero, F., Ballet, J., Decourchelle, A., et al. 2009, A\&A, 505, 157

[29] Huang, C.-Y., Park, S.-E., Pohl, M., \& Daniels, C. 2007, Astrop.Phys., 27, 429

[30] Pohl, M., Wilhelm, A., \& Telezhinsky, I. 2015, A\&A, 574, A43 